\title{
In Utero Vertical Transmission of Coronavirus Disease 2019 in a Severely III 29-week Preterm Infant
}

\author{
Suja Vinod, MD ${ }^{1}$ Emad Ghaly, MD ${ }^{1}$ \\ 1 Division of Neonatology, Department of Pediatrics, \\ $\mathrm{NYCH}+\mathrm{H} /$ Metropolitan Hospital Center, New York City, New York \\ 2 Division of Neonatology, Lewis M Fraad Department of Pediatrics, \\ $\mathrm{NYCH}+\mathrm{H} /$ Jacobi Medical Center, New York City, New York \\ ${ }^{3}$ Division of Neonatology, Department of Pediatrics, \\ $\mathrm{NYCH}+\mathrm{H} /$ Elmhurst Hospital Center, New York City, New York \\ ${ }^{4}$ Diagnostic Immunology Laboratory Wadsworth Center, New York \\ State Department of Health David Axelrod Institute for Public \\ Health, Albany, New York \\ ${ }^{5}$ Department of Microbiology, Icahn School of Medicine at Mount \\ Sinai, New York City, New York \\ ${ }^{6}$ Department of Obstetrics and Gynecology, New York Medical College, \\ $\mathrm{NYCH}+\mathrm{H} /$ Metropolitan Hospital Center, New York City, New York
}

Arpit Gupta, MD ${ }^{1}$ Yogangi Malhotra, MD $\quad$ Uday Patil, MD ${ }^{3} \quad$ Ana R. Muradas, MD ${ }^{1}$

William T. Lee, $\mathrm{PhD}^{4}$ Florian Krammer, $\mathrm{PhD}^{5}$ Fatima Amanat, $\mathrm{PhD}^{5}$ Camille A. Clare, MD, MPH

\author{
Address for correspondence Arpit Gupta, MD, FAAP, Division of \\ Neonatology, Department of Pediatrics, Metropolitan Hospital \\ Center, 1901, 1st Avenue, Room 523, New York City, NY 10029 \\ (e-mail: guptaa20@nychhc.org).
}

Am J Perinatol Rep 2020;10:e270-e274.

\begin{abstract}
Keywords

- Severe acute respiratory syndrome coronavirus 2

- coronavirus disease 2019

- preterm

- newborn

- vertical transmission

- intrauterine

Severe acute respiratory syndrome coronavirus 2 (SARS-Cov-2) or coronavirus disease 2019 (COVID-19) is currently in worldwide pandemic state with very limited data about the mode of transmission to the growing fetus. There are a few published cases of COVID-19 infection in the infants born to COVID-19 positive mothers where most of the reported cases were either mildly symptomatic with positive COVID-19 polymerase chain reaction (PCR) or had negative COVID-19 PCR raising the question of vertical transmission. We present a case of likely intrauterine transmission of COVID-19 infection in a critically ill premature infant born to a COVID-19 infected mother and describing her clinical course thus far. The clinical presentation in the infant is consistent with COVID-19 infection described so far in literature along with positive PCR, and positive COVID-19 serology: immunoglobulin $G$, immunoglobulin $M$, and immunoglobulin A.
\end{abstract}

The coronavirus disease 2019 (COVID-19) is responsible for an unprecedented epidemic in the globalized world. Though COVID-19 is known to be responsible for the severe acute respiratory syndrome coronavirus 2 (SARS-CoV-2) worldwide, the epidemiology, the clinical characteristics, and the treatment are still under investigation. The possibility of vertical transmission of SARS-CoV-2 during pregnancy has been considered but has been unproven as of yet. ${ }^{1-5}$ As per current guidelines from Centers for Disease Control, the diagnosis of COVID-19 infection relies on the positivity of the real-time reverse transcriptase (RT)-polymerase chain reaction (PCR) of the nasopharyngeal or oropharyngeal swabs, bronchioalveolar lavage, tracheal aspirates, and sputum. ${ }^{6}$ Little is known yet about the diagnosis based on the serology.

We report a case of COVID-19 infected preterm infant born to a COVID-19 infected mother. The infant has multiple RT-PCR assays positive for COVID-19, markedly abnormal inflammatory markers (known to be associated with COVID19 in published reports to date) and positive serology for COVID-19. There was no contact between the mother and the received

May 7, 2020

accepted

June 1, 2020
DOI https://doi.org/

10.1055/s-0040-1715177. ISSN 2157-6998.
Copyright $\odot 2020$ by Thieme Medical

Publishers, Inc., 333 Seventh Avenue, New York, NY 10001, USA. Tel: +1(212) 760-0888.
License terms

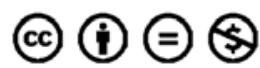


infant. All due airborne isolation precautions were taken. This is likely a case of newborn with in utero vertical transmission of COVID-19.

\section{Case Presentation}

The mother of the infant girl is a 31-year-old Gravida 2 Para 1 woman, who presented to the emergency room (ER) on April 9, 2020 with the chief complaint of fever, respiratory distress, and decreased fetal movement for 20 hours. Her fever started the day before presentation with maximum temperature of $102^{\circ} \mathrm{F}$. Her respiratory symptoms included dry cough, chest tightness, and shortness of breath. She reported developing symptoms 10 days prior to presentation. She had sick contact with the father and her 2-year-old child, both of whom were symptomatic but not tested for COVID-19 infection. Her prenatal course prior to this illness was uneventful. In the ER, she was afebrile with stable vitals except tachycardia (103 beats/minute) and no apparent respiratory distress. She was transferred to obstetrical triage for fetal assessment.

In obstetrics and gynecology triage, the fetal heart tracing was significant for decreased variability and late decelerations. The biophysical profile score was $2 / 10$. The decision was made for immediate cesarean section under spinal anesthesia. All personnel in the operating room observed airborne precautions, and the mother was given an N-95 mask during the surgery. Rupture of membranes was done at delivery, and clear fluid was noted. Infant was born limp with poor respiratory effort. She was immediately placed under the radiant warmer, and resuscitation was performed as per the Neonatal Resuscitation Program. Infant was intubated for persistent apnea at 3 minutes of life, stabilized and transferred to the neonatal intensive care unit. Apgar's scores were 3, 5, and 7 at 1, 5, and 10 minutes respectively with heart rate always above 100 beats/minute. The infant had no contact with mother after birth. Arterial cord blood was significant for mixed acidosis $\left(\mathrm{pH} 7, \mathrm{pCO}_{2} 78\right.$, and base deficit -15.2). Infant's birthweight was $1.33 \mathrm{~kg}$ (50th percentile), head circumference $28 \mathrm{~cm}$ (75th percentile) and length $41 \mathrm{~cm}$ (80th percentile).

The rapid PCR (cepheid COVID-19 PCR) done on the mother right after delivery was positive. Her intraoperative blood count was significant for leucopenia (4,500), lymphopenia $(1,400)$, and thrombocytopenia (26,000). She required 2 units each of platelets and fresh frozen plasma. Inflammatory markers sent after COVID-19 status confirmation were elevated: ferritin, $391 \mathrm{ng} / \mathrm{mL}$ (range $=15-150$ ) and procalcitonin: $0.120 \mathrm{ng} / \mathrm{mL}$ (range $=0.020-0.080$ ). A postoperative chest CT scan reported subtle peripheral opacities in bilateral lower lobes. Pathological examination of the placenta included acute and chronic villitis, intervillositis, and perivillous fibrin deposition likely representing severe placental hemorrhage consistent with disseminated intravascular coagulation. She remained stable postoperatively with no apparent respiratory distress and was discharged on day 4 of hospitalization.

In the NICU, the infant was admitted in the negative pressure room under aerosol and droplet precautions. Her Ballard maturational assessment of gestational age was con- sistent with a 29- to 30-week preterm infant. Initial respiratory presentation was consistent with severe respiratory distress syndrome with bilateral homogeneous ground glass infiltrate on chest X-ray, requiring high ventilatory support and oxygen support $\left(90 \% \mathrm{FiO}_{2}\right)$. Early surfactant (Curosurf $2.5 \mathrm{~mL} / \mathrm{kg}$ ) was administered with improvement in $\mathrm{FiO}_{2}$ requirement down to $60 \%$. Over the course of the next 24 hours, she required two additional doses of surfactant to decrease oxygen requirement to $50 \%$. The infant was started on empirical ampicillin and gentamicin. Blood culture remained negative for 5 days. She was kept nil per oral for 72 hours and was maintained on total parenteral nutrition. Expressed breast milk feeding was initiated on day 4 of life. Echocardiogram done on day 3 of life revealed atrial septal defect with left to right shunting and a small patent ductus arteriosus with bidirectional shunting. Both the ventricles were of normal size, and left ventricle was hyperdynamic. Head ultrasonography done on day 1 of life revealed bilateral grade III intraventricular hemorrhage (IVH), unrelated to her underlying thrombocytopenia, which developed on day 5 of life.

Following American Academy of Pediatrics guidelines released on April 2, 2020, a sample for COVID-19 was sent at 24 hours of life, and the test result was positive. Repeat COVID19 PCR tests sent on day 4 of life; $6,12,18$, and 25 were all positive (-Table 1 ). With respiratory symptoms, positive endotracheal PCR and persistent right-side infiltrates, she was diagnosed with pneumonia (-Fig. 1). With improvement in respiratory symptoms, she was successfully extubated on day 20. Her initial CBC was unremarkable (April 9, 2020): white blood cells (WBC) $28 \mathrm{~K}$, hematocrit 50, and platelets 224,000. Over the course of the next few days, she developed leucopenia, lymphopenia, and thrombocytopenia requiring multiple red blood cells and platelet transfusion. She had hyperglycemia for first 2 days despite giving minimal glucose infusion rate. Her initial aspartate aminotransferase and alanine aminotransferase were elevated. Inflammatory markers procalcitonin, serum lactate dehydrogenase (LDH), and serum ferritin were also significantly elevated. C-reactive protein, blood urea nitrogen, and creatinine were normal for age. The results are summarized in - Table 2.

Serology test (ELISA SARS CoV-2) was performed on the infant on April 21, day 12 and on April 27, day 18. Both the specimens were strongly positive for Immunoglobulin (Ig)G reactive with both the spike antigen and the receptor binding domain (RBD) antigen of the virus. It was also strongly positive for IgM, reactive with both the spike and the RBD. A different serology test (ARIES SARS-CoV-2 Assay, Luminex USA) was also performed on day 18 serum specimen. It showed moderate IgA reactivity with the RBD along with the strongly positive IgG and IgM reactivity for both the spike RBD and the nucleocapsid antigens. All the serology results are shown in - Fig. 2 A and $\mathbf{B}$.

\section{Discussion}

The information on in utero transmission of COVID-19 is very limited. There is no description yet on COVID-19 presentation in a premature infant. We report a severe presentation of 
Table 1 Results of coronavirus disease 2019 real-time reverse transcriptase polymerase chain reaction and serology test in the infant

\begin{tabular}{|c|c|c|c|}
\hline Date (day of life) & Laboratory/test & Specimen & PCR result \\
\hline 4/10/2020 (24 hours) & BioReference COVID-19 & Nasopharynx and oropharynx & Positive \\
\hline 4/13/2020 (day 24) & BioReference COVID -19 & Nasopharynx & Positive \\
\hline 4/15/2020 (day 6) & Cepheid COVID-19 & Nasopharynx & Positive \\
\hline 4/21/2020 (day 12) & Cepheid COVID-19 & Endotracheal aspirates & Positive \\
\hline 4/27/2020 (day 18) & Cepheid COVID-19 & Endotracheal aspirates & Positive \\
\hline 4/27/2020 (day 18) & $\begin{array}{l}\text { SARS-CoV-2 Spike (RBD) IgG Antibody } \\
\text { SARS-CoV-2 nucleocapsid IgG Antibody }\end{array}$ & Blood & Strongly positive \\
\hline 4/27/2020 (day 18) & $\begin{array}{l}\text { SARS-CoV-2 Spike (RBD) IgM Antibody } \\
\text { SARS-CoV-2 nucleocapsid IgM Antibody }\end{array}$ & Blood & Strongly positive \\
\hline 4/27/2020 (day 18) & SARS-CoV-2 Spike (RBD) IgA Antibody & Blood & Positive \\
\hline 5/2/2020 (day 25) & Cepheid COVID-19 & Nasopharynx & Positive \\
\hline
\end{tabular}

Abbreviations: COVID-19, coronavirus disease 2019; IgA, immunoglobulin A; IgG, immunoglobulin G; IgM, immunoglobulin M; PCR, polymerase chain reaction RBD, receptor binding domain; SARS-CoV-2, severe acute respiratory syndrome coronavirus 2.
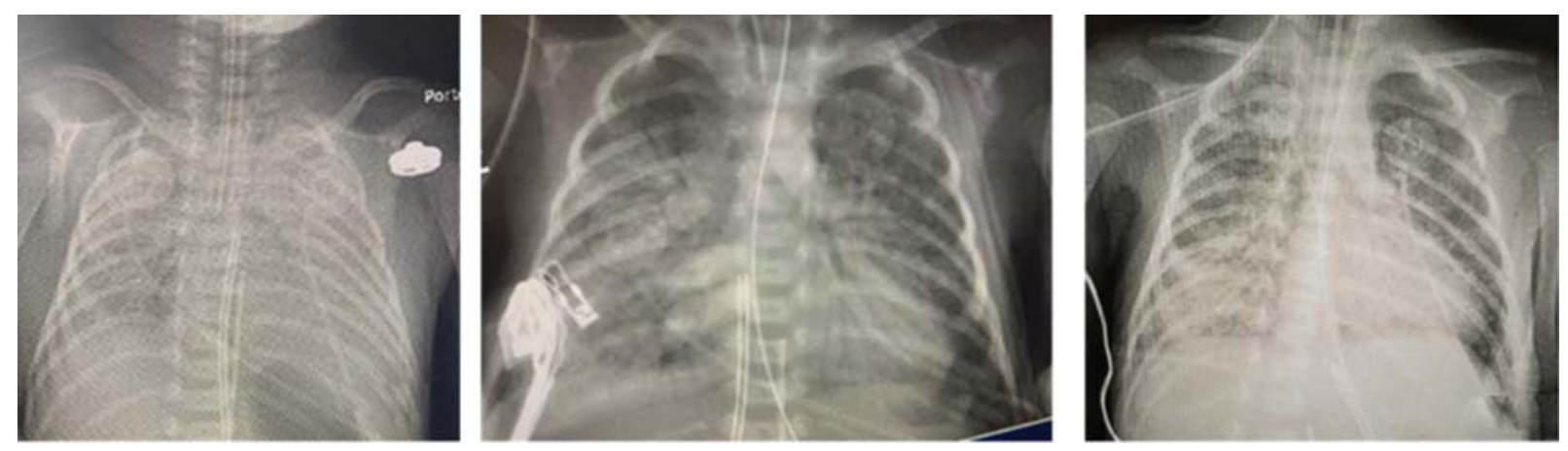

Fig. 1 Infant chest $\mathrm{X}$-ray over the period of first 12 days with persistent right-side infiltrates.

Table 2 Complete blood count in the first week of life with progressive lymphopenia, and thrombocytopenia. Elevated ferritin, lactate dehydrogenase, procalcitonin, and transaminase levels

\begin{tabular}{|c|c|c|c|c|c|c|c|c|}
\hline $\begin{array}{l}\text { Laboratory } \\
\text { test (units) }\end{array}$ & $4 / 9 / 2020$ & $4 / 10 / 2020$ & $4 / 11 / 2020$ & $4 / 12 / 2020$ & $4 / 13 / 2020$ & $4 / 14 / 2020$ & $4 / 15 / 2020$ & $4 / 16 / 2020$ \\
\hline $\begin{array}{l}\text { White cell count } \\
\left(\times 10^{9} / \mathrm{L}\right)\end{array}$ & 28.1 & 9.5 & 4.1 & 5.3 & 4.6 & 4.2 & 4 & 6.9 \\
\hline Hematocrit (\%) & 50.8 & 31.3 & 41 & 41.7 & 40.6 & 36.5 & 44.8 & 45 \\
\hline Platelet count $\left(\times 10^{9} / \mathrm{L}\right)$ & 224 & 181 & 125 & 117 & 66 & 108 & 130 & 86 \\
\hline Neutrophil (\%) & 28.9 & 63.6 & 50.8 & 61.3 & 71.2 & 53.2 & 37.6 & \\
\hline Lymphocyte (\%) & 58.7 & 20.8 & 24.6 & 31.1 & 18.2 & 31.1 & 39.5 & \\
\hline $\begin{array}{l}\text { C-reactive protein } \\
(\mathrm{mg} / \mathrm{dL})\end{array}$ & & 0.15 & & & & & & \\
\hline Procalcitonin (ng/mL) & & & & & & 0.86 & & \\
\hline Ferritin $(\mathrm{ng} / \mathrm{mL})$ & & & & & & & 1,830 & \\
\hline LDH (units/liter) & & & & & & & 1,203 & \\
\hline ALT/AST (units/liter) & & $321 / 362$ & & & & & $81 / 26$ & \\
\hline
\end{tabular}

Abbreviations: ALT, alanine aminotransferase; AST, aspartate aminotransferase; LDH, lactate dehydrogenase.

COVID-19 in a preterm infant complicated with sepsis, cytopenia, pneumonia, and severe IVH. The infant in this case was very sick from the time of birth compared with an average appropriate for gestational age of $29^{6 / 7}$ weeks preterm infant born to a relatively healthy mom. With median incubation period for COVID-19 symptoms around 5 days, no contact with mother postnatally for the first 10 days, significant clinical presentation consistent with COVID-19 in a neonate, persistently positive PCR tests, and positive serology (-Figs. 2A, B), the mode of COVID-19 transmission in this neonate is most likely to be in utero. ${ }^{4,6,7}$ Maternal consent was obtained to present the case. 
RBD IgG

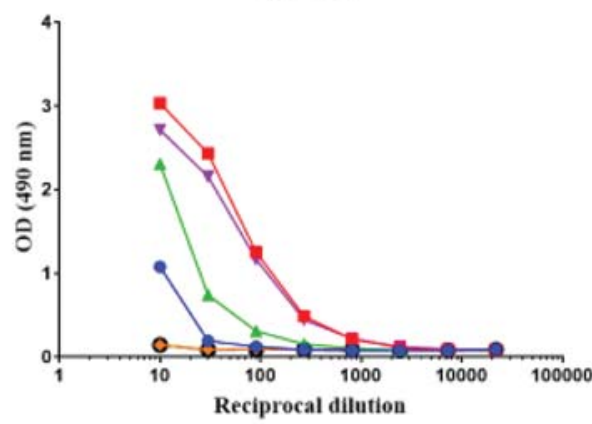

RBD IgM

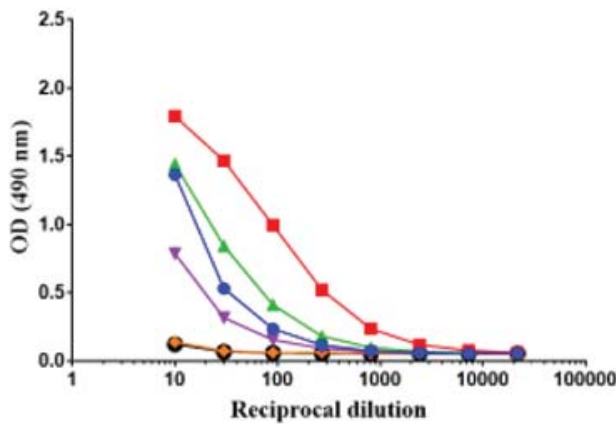

Spike IgG

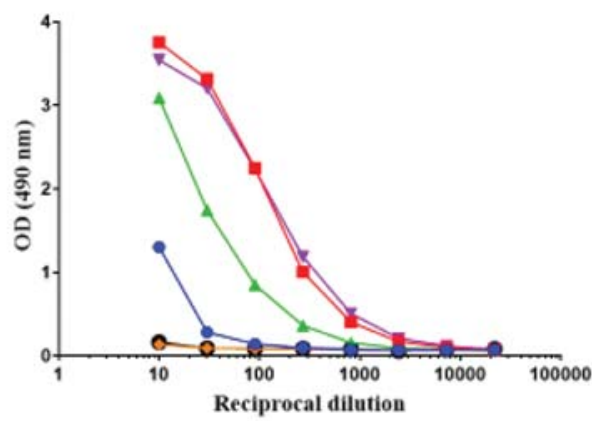

Spike IgM

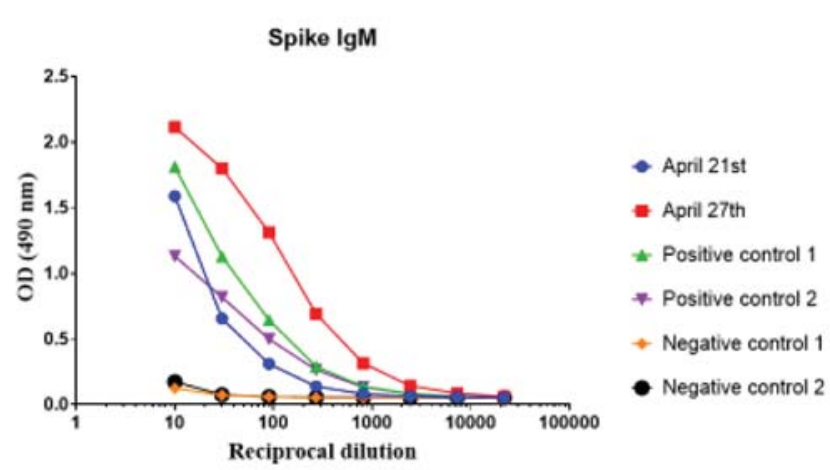

- April 21st

- April 27th

\# Positive control 1

7. Positive control 2

- Negative control 1

- Negative control 2

A

$\lg \mathrm{M}$

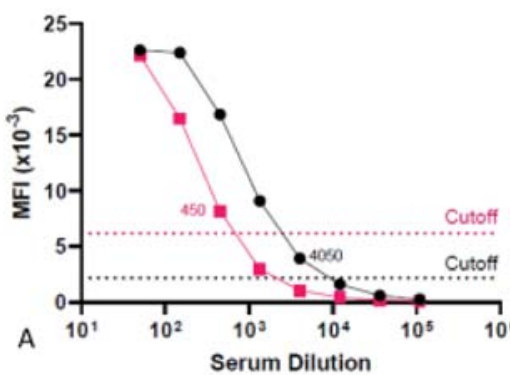

IgG $\lg A$

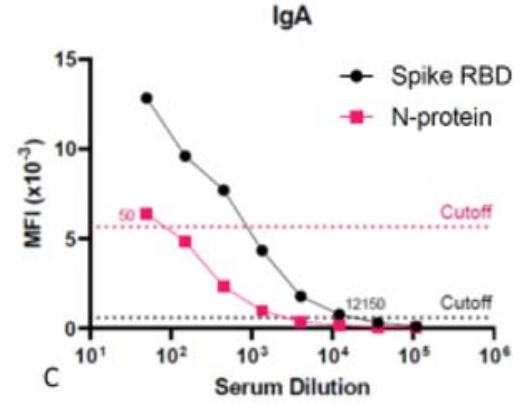

B

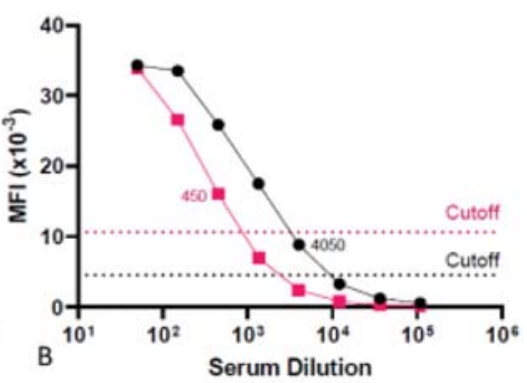

Fig. 2 Infant Serology findings. (A) ELISA SARS-CoV-2 showing reactivity of the COVID-19 antibodies at different dilutions (1:50 to 1:100,000 dilution) at day 12 and day 18 with comparison with both positive control and negative control. Antibodies were detected for spike and RBD antigens. (B) SARS-CoV-2 Assay, Luminex, at day 18 showing strongly positive immunoglobulin $G$ and immunoglobulin M for both the spike RBD and the nucleocapsid antigens. Moderate immunoglobulin A reactivity with RBD antigen is also positive. RBD, receptor binding domain; SARSCoV-2, severe acute respiratory syndrome coronavirus 2 .

Different authors in the recent past have tried to present possible cases of vertical transmission of COVID-19 infection, and not necessarily the in utero transmission. Chen et al in a study of nine confirmed COVID-19 pregnant mothers suggested no evidence of vertical transmission. ${ }^{8}$ All of the nine infants had negative COVID-19 PCR test and had minimal or mild symptoms, indicating no intrauterine exposure. Zhu et al studied 10 neonates born to mothers with COVID-19 2019-nCoV pneumonia and found that "perinatal 2019-nCoV infection may have adverse effects on newborns, causing problems such as fetal distress, premature labor, respiratory distress, thrombocytopenia accompanied by abnormal liver

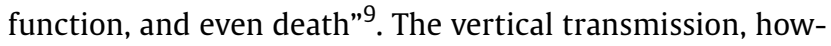
ever, could not be proved. Six out of ten infants in their study were mild to moderately symptomatic with one mortality due to multiorgan failure with no clear diagnosis and all the infants in the study had negative COVID-19 PCR.

In two research letters published in JAMA Pediatrics, Zeng et $\mathrm{al}^{2}$ and Dong et $\mathrm{al}^{3}$ reported cases of possible vertical transmission of COVID-19 infection based on the presence of IgM antibody in the infant's serum. However, all of the infants in their respective studies had negative COVID-19 PCR test. This raised questions over the possibility of in utero transmission as cautioned by Kimberly and Stagno in the accompanying editorial in JAMA Pediatrics. ${ }^{10}$ The caution was in terms of lack of clarity of the medical literature on the kinetics of COVID-19 specific antibody production, half-life, potential cross-reactivity with other antigens, and other testing challenges. 
Similar to SARS-CoV, antibodies are produced in response to different SARS-CoV-2 antigens, one is outer spike receptor binding domain and the other is inner nucleocapsid. ${ }^{11}$ As per the $\mathrm{CDC}$, it may take up to 1 to 3 weeks, or longer postexposure before the antibody is detected in serum. ${ }^{12}$ The exact information on the kinetics of COVID-19 is still unclear. IgG antibodies are the only class of immunoglobulins known to crossover to the fetus and are present at birth. ${ }^{13}$ IgM antibodies are always neonatal in origin and are detectable usually 1 to 4 weeks postexposure depending upon the disease. ${ }^{13,14}$ IgA antibodies are primarily secretory in nature and are the predominant immunoglobulin isotype present on mucosal surfaces. Most investigators report the initial detection of IgA in mucosal secretions between 1 week and 2 months with peak levels between 4 to 6 weeks of age. ${ }^{14}$ Several studies have suggested presence of IgA antibodies in the newborn period to be primarily of fetal origin. ${ }^{15,16}$

The infant's sera in this case on day 13 was strongly positive for both $\operatorname{IgG}$ and IgM with a further increase in reactivity on day 18. Day 18 serology (Luminex) showed moderate IgA alongside strongly positive IgG and IgM. Both the serology tests (SARS-CoV-2 ELISA, and Luminex) are Food and Drug Administration approved, and carry specificity of $100 \%$ with a sensitivity in high 90s. ${ }^{17,18}$ Placental histopathology findings of villitis, intervillositis, and hemorrhage in the case were also consistent with COVID-19 infection as described recently by Shanes et al. ${ }^{19}$ Based on literature as described above, we can safely presume that the exposure to COVID-19 in the infant is most likely intrauterine or preceding the delivery.

Limitation of this report is the absence of placental or cord blood for COVID-19 antigen and antibody detection. However, the data on the utility of 2019-nCoV real-time RT-PCR in amniotic fluid, blood, and placenta are very limited. Serologic testing for IgG and IgM is still in the experimental phase and not available for routine diagnosis. Little is also known on the time frame of antibody appearance and the half-life of COVID19 specific $\operatorname{Ig} G$ and IgM antibodies especially in infants.

\section{Conclusion}

COVID-19 is responsible for an unprecedented epidemic in the globalized world, and the infant and childhood infection is still not well known. Though the vertical intrauterine transmission is not proven in the literature yet, we report a case of a vertical neonatal COVID-19 infection. With clinical characteristics, laboratory and serology findings compatible with SARS$\mathrm{CoV}-2$, this appears be a case of in utero transmission. Further studies looking at in utero transmission are recommended.

\section{Conflict of Interest}

None declared.

\section{References}

1 Zeng L, Xia S, Yuan W, et al. Neonatal early-onset infection with SARSCoV-2 in 33 neonates born to mothers with COVID-19 in Wuhan, China. JAMA Pediatr 2020 Doi: 10.1001/jamapediatrics.2020.0878
2 Zeng H, Xu C, Fan J, et al. Antibodies in infants born to mothers with COVID-19 pneumonia. JAMA 2020 Doi: 10.1001/jama.2020.4861

3 Dong L, Tian J, He S, et al. Possible vertical transmission of SARSCoV-2 from an infected mother to her newborn. JAMA 2020 Doi: 10.1001/jama.2020.4621

4 Puopolo K, Hudak M, Kimberlin D, Cummings J. Initial guidance: management of infants born to mothers with COVID-19. Available at: https://www.tn.gov/content/dam/tn/health/documents/cedep/ novel-coronavirus/AAP_COVID-19-Initial-Newborn-Guidance.pdf. Accessed 2, 2020

5 Karimi-Zarchi M, Neamatzadeh H, Dastgheib SA, et al. Vertical transmission of coronavirus disease 19 (COVID-19) from infected pregnant mothers to neonates: a review. Fetal Pediatr Pathol 2020;39(03):246-250

6 Centers for Disease Control and Prevention. Interim clinical guidance for management of patients with confirmed 2019 novel coronavirus (2019-nCoV) infection. Available at: https://www. cdc.gov/coronavirus/2019-ncov/hcp/clinical-guidance-management-patients.html. Accessed February 12, 2020

7 Lauer SA, Grantz KH, Bi Q et al. The incubation period of coronavirus disease 2019 (COVID-19) from publicly reported confirmed cases: estimation and application. Ann Intern Med 2020;172(09):577-582

8 Chen Y, Peng H, Wang L, et al. Infants born to mothers with a new coronavirus (COVID-19). Front Pediatr 2020;8:104 Doi: 10.3389/ fped.2020.00104

9 Zhu H, Wang L, Fang C, et al. Clinical analysis of 10 neonates born to mothers with 2019-nCoV pneumonia. Transl Pediatr 2020;9 (01):51-60

10 Kimberlin DW, Stagno S. Can SARS-CoV-2 infection be acquired in utero?: more definitive evidence is needed JAMA 2020 Doi: 10.1001/jama.2020.4868

11 Fehr AR, Perlman S. 2015Coronaviruses: an overview of their replication and pathogenesis. In: Maier $\mathrm{H}$, Bickerton E, Britton $\mathrm{P}$, eds. Coronaviruses: Methods in Molecular Biology, vol 1282. New York City, NY: Humana Press Doi: 10.1007/978-1-4939-2438-7_1

12 Centers for Disease Control and Prevention. Antibody testing for COVID-19. Available at: https://www.cdc.gov/coronavirus/2019ncov/lab/resources/antibody-tests.html. Accessed May 28, 2020

13 Shen C, Xu H, Liu D, Veazey RS, Wang X. Development of serum antibodies during early infancy in rhesus macaques: implications for humoral immune responses to vaccination at birth. Vaccine 2014;32(41):5337-5342

14 Gleeson M, Cripps AW. Development of mucosal immunity in the first year of life and relationship to sudden infant death syndrome. FEMS Immunol Med Microbiol 2004;42(01):21-33

15 Ben-Hur H, Gurevich P, Huszar M, et al. Immunoglobulin A in the epithelium of the respiratory tract and intrahepatic bile ducts of fetuses and newborns with pneumonia and sepsis. Hum Antibodies 1997;8(03):119-123

16 Borte S, Janzi M, Pan-Hammarström Q et al. Placental transfer of maternally-derived IgA precludes the use of guthrie card eluates as a screening tool for primary immunodeficiency diseases. PLoS One 2012;7(08):e43419

17 Rao A, Goldstein DY, Wolk DM, Wolf LA. Development and evaluation of two SARS-CoV-2 RT-PCR laboratory developed tests on the ARIES ${ }^{\circledR}$ automated, sample-to-answer, real-time PCR system. . Available at: https://www.captodayonline.com/LuminexSARSCoV219.pdf. Accessed March 12, 2020

18 Amanat F, Nguyen T, Chromikova V, et al. A serological assay to detect SARS-CoV-2 seroconversion in humans. Available at: https://www. medrxiv.org/content/10.1101/2020.03.17.20037713v2. Accessed April 16, 2020

19 Shanes ED, Mithal LB, Otero S, Azad HA, Miller ES, Goldstein JA. Placental Pathology in COVID-19. Am J Clin Pathol 2020;154(01): 23-32 